\title{
Manifolds with small Heegaard Floer ranks
}

\author{
MATTHEW HEDDEN \\ YI NI
}

\begin{abstract}
We show that the only irreducible three-manifold with positive first Betti number and Heegaard Floer homology of rank two is homeomorphic to zero-framed surgery on the trefoil. We classify links whose branched double cover gives rise to this manifold. Together with a spectral sequence from Khovanov homology to the Floer homology of the branched double cover, our results show that Khovanov homology detects the unknot if and only if it detects the two component unlink.
\end{abstract}

57M27; 57M25

\section{Introduction}

In a sequence of papers, Ozsváth and Szabó defined invariants for a wide variety of topological and geometric objects in low dimensions, including three- and fourmanifolds, knots and links and contact structures $[29 ; 28 ; 27 ; 35 ; 30]$. These invariants proved to be quite powerful, with striking applications to questions in Dehn surgery, contact and symplectic geometry, knot concordance and questions about unknotting numbers (to name only a few).

The Ozsváth-Szabó invariants are particularly suited to understand homologically essential surfaces embedded in three-manifolds. In the context of knots, they demonstrated this by the theorem that knot Floer homology detects the Seifert genus of a knot [26]. More generally, Ozsváth and Szabó showed the Floer invariants capture the Thurston norm on the homology of link complements and closed three-manifolds [26; 36]. These theorems have the immediate consequence that knot Floer homology detects the unknot, as this is the only knot of Seifert genus zero.

In addition to the Thurston norm of a homology class $\alpha \in H_{2}(Y, \partial Y)$, the OzsváthSzabó invariants answer the subtler question of when a knot complement or closed three-manifold fibers over the circle with fiber an embedded surface $\Sigma$, whose homology class equals $\alpha$; see Ghiggini [9], Ni [23; 24] and Ai and Ni [1]. For knots, this again has a beautiful corollary by Ghiggini [9], namely that knot Floer homology detects the trefoil and figure eight knots. This follows from the well-known fact that these are the 
only fibered knots with Seifert genus one (together with an easy computation showing their Floer homologies are different).

In this article we prove a similar theorem in the context of closed three-manifolds. Namely, we determine which irreducible three-manifolds with positive first Betti number have rank 2 Floer homology; there is only one.

Theorem 1.1 Suppose $Y$ is a closed oriented irreducible 3-manifold which satisfies $b_{1}(Y)>0$. If $\operatorname{rank} \widehat{H F}(Y)=2$, then $Y$ is homeomorphic (via a possibly orientationreversing map) to $S_{0}^{3}\left(3_{1}\right)$, the manifold obtained by zero surgery on the trefoil.

Removing the irreducibility requirement, we obtain the following:

Corollary 1.2 Suppose $Y$ is a closed oriented 3-manifold with $b_{1}(Y)>0$. If the rank of $\widehat{H F}(Y)$ is 2 , then $Y=Y^{\prime} \# Z$, where $Y^{\prime}$ is either $\pm S_{0}^{3}\left(3_{1}\right)$ or $S^{1} \times S^{2}$ and $Z$ is an integer homology sphere satisfying $\widehat{H F}(Z) \cong \mathbb{Z}$.

In addition to our interest in the theorem as it pertains to understanding the power of Heegaard Floer homology, we can use it to gain new insight into the mysterious world of quantum invariants. Indeed, the existence of a spectral sequence from the Khovanov homology of a link to the Heegaard Floer homology of its branched double cover given by Ozsváth and Szabó [32] allows us to prove the following theorem. To state it, let $\mathbb{F}=\mathbb{Z} / 2 \mathbb{Z}$.

Theorem 1.3 Suppose $L \subset S^{3}$ is a link with $\operatorname{det}(L)=0$. Then

$$
\operatorname{rank}_{\mathbb{F}} K h(L ; \mathbb{F})>4
$$

unless $L$ is isotopic to a two-component split link $L=K_{1} \sqcup K_{2}$ satisfying

$$
\operatorname{rank}_{\mathbb{F}} K h\left(K_{i} ; \mathbb{F}\right)=2, \quad i=1,2 .
$$

Corollary 1.4 Khovanov homology detects the unknot if and only if it detects the two-component unlink.

Remark Recently, Kronheimer and Mrowka proved that Khovanov homology detects the unknot [18]. Hence Khovanov homology detects the two-component unlink. It is worth pointing out that our result does not directly follow from Kronheimer and Mrowka's argument. 
This theorem should be compared with the results of Thistlethwaite [40] and Eliahou, Kauffman and Thistlethwaite [6] which provide examples of two (or more) component links with Jones polynomial equal to that of the unlink. By construction these links are nonsplit, so our result implies that they are distinguished from the unlink by their Khovanov homology. This highlights the strength of Khovanov homology over its Euler characteristic and indicates that the question of whether Khovanov homology detects the unknot may be of a much different nature than the corresponding question for the Jones polynomial.

The proof of Theorem 1.1 begins by using the Thurston norm detection of Floer homology to show the rank assumption implies that the manifold contains a homologically essential torus. The ability of Floer homology to detect fibering then shows that this torus is the fiber in a surface bundle over the circle. Lower bounds on the rank of Floer homology in terms of $H_{1}(Y ; \mathbb{Z})$, together with a simple analysis of this group for torus fibrations, show that the manifold is zero surgery on the trefoil or figure eight. The latter manifold, however, has Floer homology of rank four by direct calculation.

Theorem 1.3 follows from the spectral sequence from Khovanov homology to the Floer homology of the branched double cover mentioned above. The main work in this step is to understand which links in the three-sphere have $S_{0}^{3}\left(3_{1}\right)$ as their branched double cover. We classify such links (there are essentially only two) in Section 4 by a geometric argument based on the fact that $S_{0}^{3}\left(3_{1}\right)$ admits a Euclidean geometry.

We conclude by remarking that the question of whether Khovanov homology detects the unknot could likely be understood through Heegaard Floer homology if the manifolds, $Z$, appearing in Corollary 1.2 could be appropriately classified. Indeed, the question of which integer homology three-spheres have rank one Floer homology (the so-called homology sphere L-spaces) is interesting for a variety of reasons. Even with our limited understanding of homology sphere L-spaces, one can gain useful information about Khovanov homology. For instance, one can easily show that the Khovanov homology of the two-cable (and many other satellite operations) detects the unknot; see Hedden [12] and Hedden and Watson [13]. See also Eftekhary [5] and Grigsby and Wehrli [10] for related results.

Acknowledgements We are very grateful to Peter Ozsváth for a helpful conversation about the Universal Coefficients Theorem. We thank Yanki Lekili for helpful conversations. We also thank Chuck Livingston and Alan Edmonds for their interest in this work. The first author was partially supported by NSF grant number DMS-0706979 and thanks the Indiana University math department for their hospitality during an extended visit in which some of this work was completed. The second author was partially supported by an AIM Five-Year Fellowship and NSF grant number DMS-0805807. 


\section{Preliminaries}

In this section we recall some necessary background on the Heegaard Floer and Khovanov homology theories, respectively. Our purpose is mainly to establish notation and collect results which will be used in the subsequent sections. For the unfamiliar reader, we refer to Ozsváth and Szabó [34; 31] for an introduction to Ozsváth-Szabó theory and Khovanov [16] and Bar-Natan [3] for material on Khovanov homology.

\subsection{Twisted Heegaard Floer homology}

Heegaard Floer homology (or Ozsváth-Szabó homology) assigns chain complexes to a $\operatorname{Spin}^{c}$ three-manifold, $(Y, \mathfrak{s})$. One can import the general construction of homology with twisted coefficients (see, for instance Hatcher [11]) into this theory in a variety of useful ways, which we now recall.

The input for the theory is an admissible pointed Heegaard diagram

$$
(\Sigma, \boldsymbol{\alpha}, \boldsymbol{\beta}, z)
$$

for $(Y, \mathfrak{s})$. Taking the symmetric product of the diagram, one arrives at the $2 g-$ dimensional symplectic manifold $\operatorname{Sym}^{g}(\Sigma)$, together with two Lagrangian submanifolds $\mathbb{T}_{\alpha}, \mathbb{T}_{\beta}$, and a distinguished hypersurface, $V_{z}$, which arise from the attaching curves and basepoint, respectively (see Ozsváth and Szabó [29] for more details, specifically Sections 2 and 4).

The most general construction of Heegaard Floer homology with twisted coefficients defines a chain complex $\underline{C F^{\infty}}(Y, \mathfrak{s})$ which is freely generated over the ring $\mathbb{Z}\left[U, U^{-1}\right] \otimes_{\mathbb{Z}}$ $\mathbb{Z}\left[H^{1}(Y ; \mathbb{Z})\right]$ by intersection points $\mathbf{x} \in \mathbb{T}_{\alpha} \cap \mathbb{T}_{\beta}$ whose associated Spin ${ }^{c}$ structure corresponds to $\mathfrak{s}$. Here $H^{1}(Y ; \mathbb{Z})$ is the first singular cohomology of $Y$, and $U$ is a formal variable of degree -2 .

Given $\mathbf{x}, \mathbf{y} \in \mathbb{T}_{\alpha} \cap \mathbb{T}_{\beta}$, let $\pi_{2}(\mathbf{x}, \mathbf{y})$ denote the set of homotopy classes of Whitney disks connecting $\mathbf{x}$ to $\mathbf{y}$. The twisted coefficient ring is a reflection of the fact that $\pi_{2}(\mathbf{x}, \mathbf{y}) \cong \mathbb{Z} \oplus H^{1}(Y ; \mathbb{Z}) .{ }^{1}$ Pick an additive assignment $A: \pi_{2}(\mathbf{x}, \mathbf{y}) \rightarrow H^{1}(Y)$ in the sense of Definition 2.12 of [29], and define an endomorphism

$$
\underline{\partial}: \underline{C F}^{\infty}(Y, \mathfrak{s}) \rightarrow \underline{C F}^{\infty}(Y, \mathfrak{s}),
$$

\footnotetext{
${ }^{1}$ To identify this with the standard construction of homology with twisted coefficients, observe that $\pi_{2}(\mathbf{x}, \mathbf{y})$ is the fundamental group of the configuration space of paths in $\operatorname{Sym}^{g}(\Sigma)$ from $\mathbb{T}_{\alpha}$ to $\mathbb{T}_{\beta}$. Heuristically, Heegaard Floer homology is the Morse homology of this configuration space with respect to a specific action functional.
} 
by the formula:

$$
\underline{\partial \mathbf{x}}=\sum_{\mathbf{y} \in \mathbb{T}_{\alpha} \cap \mathbb{T}_{\beta}} \sum_{\left\{\phi \in \pi_{2}(\mathbf{x}, \mathbf{y}) \mid \mu(\phi)=1\right\}} \#(\widehat{\mathcal{M}}(\phi)) U^{n_{z}(\phi)} \otimes e^{A(\phi)} \cdot \mathbf{y},
$$

where $\widehat{\mathcal{M}}(\phi)$ is the quotient of the moduli space of unparametrized $J$-holomorphic disks representing the homotopy class $\phi, \mu(\phi)$ is the Maslov index, and $n_{z}(\phi)=$ $\# \phi \cap V_{z}$ is the algebraic intersection of $\phi$ with the hypersurface. Here, we have denoted elements in $\mathbb{Z}\left[H^{1}(Y)\right]$ in the typical exponential notation. Gromov compactness for $J$-holomorphic curves ensures that $\underline{\partial} \circ \underline{\partial}=0$, and we denote the homology of the resulting chain complex by $\underline{H F}^{\infty}(Y, \mathfrak{s})$. The results of [29;28] indicate that these groups depend only on the $\operatorname{Spin}^{c}$-diffeomorphism type of the pair $(Y, \mathfrak{s})$. We refer the reader to the aforementioned papers for more details. Section 8 of [28], in particular, introduces this notion of twisted coefficients.

We could alternatively take the chain complexes to be generated over either of the rings

$$
U \cdot \mathbb{Z}[U] \otimes \mathbb{Z}\left[H^{1}(Y)\right] \quad \mathbb{Z}\left[U, U^{-1}\right] / U \cdot \mathbb{Z}[U] \otimes \mathbb{Z}\left[H^{1}(Y)\right] .
$$

We denote the resulting complexes by $\underline{C F}^{-}(Y, \mathfrak{s}), \underline{C F}^{+}(Y, \mathfrak{s})$, respectively. Positivity of intersections for $J$-holomorphic curves ensures that this is well-defined and, moreover, that we have a short exact sequence

$$
0 \rightarrow \underline{C F}^{-}(Y, \mathfrak{s}) \rightarrow \underline{C F}^{\infty}(Y, \mathfrak{s}) \rightarrow \underline{C F}^{+}(Y, \mathfrak{s}) \rightarrow 0
$$

(with corresponding long exact sequence of homology). We can also define a complex $\widehat{C F}(Y, \mathfrak{s})$ by the exact sequence

$$
0 \rightarrow \underline{\widehat{C F}}(Y, \mathfrak{s}) \rightarrow \underline{C F^{+}}(Y, \mathfrak{s}) \stackrel{\cdot U}{\rightarrow} \underline{C F}^{+}(Y, \mathfrak{s}) \rightarrow 0 .
$$

The homology of the various complexes are invariants of the pair $(Y, \mathfrak{s})$ and are denoted $\underline{H F^{+}}, \underline{H F^{-}}, \underline{\widehat{H F}}$.

Note $\mathbb{Z}\left[U, U^{-1}\right] \otimes \mathbb{Z}\left[H^{1}(Y)\right]$ is naturally a $\mathbb{Z}\left[H^{1}(Y)\right]$ module (by letting $\mathbb{Z}\left[H^{1}(Y)\right]$ act trivially on $\left.\mathbb{Z}\left[U, U^{-1}\right]\right)$. Thus, for any $\mathbb{Z}\left[H^{1}(Y)\right]$-module, $M$, it makes sense to consider Heegaard Floer homology with coefficients in $M$. By definition, these are the groups

$$
\underline{H F}^{\circ}(Y, \mathfrak{s} ; M):=H_{*}\left(\underline{C F^{\circ}}(Y, \mathfrak{s}) \otimes_{\mathbb{Z}\left[H^{1}(Y)\right]} M\right),
$$

where $\underline{C F^{\circ}}$ denotes any of the chain complexes $(\hat{ \pm}, \infty)$ considered above.

We will be interested in $\mathbb{Z}\left[H^{1}(Y)\right]$-modules which focus attention on the part of $H^{1}(Y)$ which pairs nontrivially with certain 1-dimensional homology classes. More 
precisely, given $\omega \in H_{1}(Y)$, we consider the ring $R=\mathbb{Q}\left[T, T^{-1}\right]$ as a $\mathbb{Z}\left[H^{1}(Y)\right]$ module by defining the action

$$
e^{\gamma} \cdot 1=T^{\gamma(\omega)}
$$

where $1 \in \mathbb{Q}\left[T, T^{-1}\right], e^{\gamma} \in \mathbb{Z}\left[H^{1}(Y)\right]$, and $\gamma(\omega)$ is the natural pairing between cohomology and homology. We denote chain complexes with coefficients in $R$ by

$$
\underline{C F}^{\circ}\left(Y, \mathfrak{s} ; R_{\omega}\right):=\underline{C F^{\circ}}(Y, \mathfrak{s}) \otimes_{\mathbb{Z}\left[H^{1}(Y)\right]} R,
$$

and refer to them as the $\omega$-twisted Heegaard Floer chain complexes. We can also complete this coefficient ring in a particularly useful way. Define an $R$-module by

$$
\Lambda=\left\{\sum_{r \in \mathbb{R}} a_{r} T^{r} \mid a_{r} \in \mathbb{R}, \#\left\{a_{r} \mid a_{r} \neq 0, r \leq c\right\}<\infty \text { for any } c \in \mathbb{R}\right\},
$$

where $R$ acts on $\Lambda$ by polynomial multiplication. We refer to $\Lambda$ as the universal Novikov ring, and the corresponding chain complexes

$$
\underline{C F^{\circ}}\left(Y, \mathfrak{s} ; \Lambda_{\omega}\right):=\underline{C F^{\circ}}\left(Y, \mathfrak{s} ; R_{\omega}\right) \otimes_{R} \Lambda
$$

as Heegaard Floer chain complexes with $\omega$-twisted Novikov coefficients or, following [15], as the $\omega$-perturbed Floer homology. Observe that $\Lambda$ is a field.

Note that we can disregard the twisting altogether by letting $R$ act trivially on $\mathbb{Q}$ (or any other ring, eg $\mathbb{Z}$, or $\mathbb{Z} / 2 \mathbb{Z}$ ) by the rule $T \cdot a=a$, where $a \in \mathbb{Q}$ (with corresponding trivial action on any other ring). The resulting chain complexes

$$
C F^{\circ}(Y, \mathfrak{s} ; \mathbb{Q}):=\underline{C F^{\circ}}\left(Y, \mathfrak{s} ; R_{\omega}\right) \otimes_{R} \mathbb{Q}
$$

are the ordinary (untwisted) Heegaard Floer chain complexes with coefficients in $\mathbb{Q}$. Throughout, we will adopt the notation that $H F^{\circ}(Y, \mathfrak{s}):=H F^{\circ}(Y, \mathfrak{s} ; \mathbb{Z})$, ie in the untwisted world we use $\mathbb{Z}$ coefficients unless otherwise specified.

Finally, given $[F] \in H_{2}(Y ; \mathbb{Z})$, we can consider the direct sum of chain complexes corresponding to $\operatorname{Spin}^{c}$ structures on $Y$ whose first Chern class evaluates on $[F]$ as a specified integer. In this case, we adopt the notation:

$$
C F^{\circ}(Y,[F], i):=\bigoplus_{\left\{\mathfrak{s} \in \operatorname{Spin}^{c}(Y) \mid\left\langle c_{1}(\mathfrak{s}),[F]\right\rangle=2 i\right\}} C F^{\circ}(Y, \mathfrak{s}),
$$

with corresponding notation for homology groups and the various coefficient rings described above. 


\subsection{Nontriviality theorems}

In this subsection, we collect a few key theorems concerning nontriviality of Floer homology. We first state a generalization of Ozsváth and Szabó's theorem that Floer homology detects the Thurston norm of a closed three-manifold. To do this recall that the complexity of a closed surface, $F$, is the quantity

$$
x(F)=\sum_{F_{i} \subset F} \max \left\{0,-\chi\left(F_{i}\right)\right\},
$$

where the sum is taken over all connected components of $F$ and $\chi$ is the Euler characteristic. A homologically nontrivial surface, $F$, in an irreducible three-manifold is called taut if it minimizes complexity amongst all embedded surfaces whose associated homology class is equal to $[F] \in H_{2}(Y ; \mathbb{Z})$. We have the following:

Theorem 2.1 [22, Theorem 3.6] Suppose $Y$ is a closed irreducible 3-manifold, and $F$ is a taut surface in $Y$. Then there exists a nonempty open set $U \subset H_{1}(Y ; \mathbb{R})$, such that for any $\omega \in U$,

$$
\underline{H F}^{+}\left(Y,[F], \frac{1}{2} x(F) ; \Lambda_{\omega}\right) \neq 0, \quad \underline{\widehat{H F}}\left(Y,[F], \frac{1}{2} x(F) ; \Lambda_{\omega}\right) \neq 0 .
$$

This implies the following for the homology with untwisted coefficients:

Theorem 2.2 Suppose $Y$ is a closed 3-manifold, $F$ is a taut surface in $Y$. Then

$$
\widehat{H F}\left(Y,[F], \frac{1}{2} x(F)\right) \otimes \mathbb{Q} \neq 0 .
$$

Proof In Theorem 2.1, we can choose $\omega$ be a rational homology class. Since $\underline{H F}^{+}\left(Y ; \Lambda_{\omega}\right) \cong \underline{H F}^{+}\left(Y ; \Lambda_{k \omega}\right)$ for any nonzero $k$, we may assume $\omega$ is an integral homology class.

Since $R=\mathbb{Q}\left[T, T^{-1}\right]$ is a PID, the universal coefficients theorem [39] implies

$$
\underline{\widehat{H F}}\left(-; \Lambda_{\omega}\right) \cong\left(\underline{\widehat{H F}}\left(-; R_{\omega}\right) \otimes_{R} \Lambda\right) \oplus \operatorname{Tor}_{R}\left(\underline{\widehat{H F}}\left(-; R_{\omega}\right), \Lambda\right) .
$$

Since $\Lambda$ is $R$ torsion-free the Tor term vanishes. The nonvanishing result for the left-hand side (Theorem 2.1) then implies that $\underline{\widehat{H F}}\left(Y,[F], \frac{1}{2} x(F) ; R_{\omega}\right) \otimes_{R} \Lambda \neq 0$, and hence $\underline{\widehat{H F}}\left(Y,[F], \frac{1}{2} x(F) ; R_{\omega}\right)$ has positive rank as an $R$-module. Applying the universal coefficients theorem again,

$$
\widehat{H F}(-; \mathbb{Q}) \cong\left(\underline{\widehat{H F}}\left(-; R_{\omega}\right) \otimes_{R} \mathbb{Q}\right) \oplus \operatorname{Tor}_{R}\left(\underline{\widehat{H F}}\left(-; R_{\omega}\right), \mathbb{Q}\right),
$$


we find that the untwisted Floer homology with $\mathbb{Q}$ coefficients is nontrivial. On the other hand, this latter group is isomorphic to

$$
\widehat{H F}(-; \mathbb{Q}) \cong\left(\widehat{H F}(-) \otimes_{\mathbb{Z}} \mathbb{Q}\right) \oplus \operatorname{Tor}_{\mathbb{Z}}(\widehat{H F}(-), \mathbb{Q}) .
$$

The Tor term vanishes since $\mathbb{Q}$ has no $\mathbb{Z}$ torsion. This yields the result.

Lemma 2.3 If $H F^{\infty}(Y, \mathfrak{s})$ contains $k$ copies of $\mathbb{Z}\left[U, U^{-1}\right]$ as direct summands, then

$$
\operatorname{rank} \widehat{H F}(Y, \mathfrak{s}) \geq k \text {. }
$$

Proof This follows easily from the two exact sequences

$$
\begin{gathered}
\cdots \rightarrow H F^{-} \rightarrow H F^{\infty} \rightarrow H F^{+} \rightarrow \cdots \\
\cdots \rightarrow H F^{+} \rightarrow H F^{+} \rightarrow \widehat{H F} \rightarrow \cdots
\end{gathered}
$$

and the fact that $H F_{i}^{-}=0$ when $i$ is sufficiently large.

\subsection{Khovanov homology}

To a link $L \subset S^{3}$, Khovanov homology associates a collection of bigraded abelian groups, $K h_{i, j}(L)$ [16]. The graded Euler characteristic of these groups is the Jones polynomial, in the sense that

$$
\left(q+q^{-1}\right) \cdot J_{L}\left(q^{2}\right)=\sum_{j}\left(\sum_{i}(-1)^{i} \operatorname{rank} K h_{i, j}(L)\right) \cdot q^{j},
$$

where $J_{L}(q)$ is the Jones polynomial, and "rank" is taken to mean the rank as a $\mathbb{Z}-$ module. For the present purpose, it will not be necessary to explain the precise details of Khovanov's construction. It suffices to say that the groups arise as the (co)homology groups of a bigraded (co)chain complex, $C K h_{i, j}(D)$, associated to a link diagram, $D$. The complex is obtained by applying a $(1+1)$ dimensional topological quantum field theory to the cube of complete resolutions of $D$. The $i$-grading is the cohomological grading ie the differential increases this grading by one, while the $j$-grading is the so-called quantum grading (corresponding to the variable in the Jones polynomial). In the original treatment, the differential preserved the $j$-grading. Lee considered a perturbation of this differential which does not preserve the $j$-grading, but instead makes $C K h_{i, j}(L)$ into a complex filtered by $j$ [19]. This perturbation was useful for several purposes, most notably in Rasmussen's combinatorial proof of Milnor's conjecture on the unknotting number of torus knots [37]. A consequence of Lee's work which will be useful for us is the following: 
Theorem 2.4 [19] Let $L \subset S^{3}$ be a link of $|L|$ components. Then

$$
\operatorname{rank} K h(L) \geq 2^{|L|} \text {. }
$$

The main feature of Khovanov homology which we use is a connection with the Heegaard Floer invariants.

Theorem 2.5 [32, Theorem 1.1] Let $L \subset S^{3}$ be a link. There is a spectral sequence whose $E_{2}$ term consists of $\widetilde{K h}(\bar{L} ; \mathbb{Z} / 2 \mathbb{Z})$, Khovanov's reduced homology of the mirror of $L$, and which converges to $\widehat{H F}(\Sigma(L) ; \mathbb{Z} / 2 \mathbb{Z})$, the Heegaard Floer homology of the branched double cover of $L$.

The reduced Khovanov homology is a variant of Khovanov homology defined via a chain complex, $\widetilde{C K h}$, which has half the rank (taken in any coefficient ring) of $C K h(L)$. In the case of $\mathbb{Z} / 2 \mathbb{Z}$ coefficients, there is little difference between the reduced and ordinary theories. Indeed,

$$
K h(L ; \mathbb{Z} / 2 \mathbb{Z}) \cong \widetilde{K h}(L ; \mathbb{Z} / 2 \mathbb{Z}) \otimes V,
$$

where $V$ is the rank 2 vector space over $\mathbb{Z} / 2 \mathbb{Z}$ obtained as the Khovanov homology of the unknot (see, for instance, Shumakovitch [38]).

Packaging all of this, the result we use is the following:

Proposition 2.6 Let $L \subset S^{3}$ be a link of $|L|$ components and $\Sigma(L)$ denote its branched double cover.

$$
\operatorname{rank}_{\mathbb{Z} / 2 \mathbb{Z}} K h(L ; \mathbb{Z} / 2 \mathbb{Z}) \geq 2 \cdot \operatorname{rank}_{\mathbb{Z}} \widehat{H F}(\Sigma(L))
$$

Proof Theorem 2.5 immediately yields

$$
\operatorname{rank}_{\mathbb{Z} / 2 \mathbb{Z}} \widetilde{K h}(\bar{L} ; \mathbb{Z} / 2 \mathbb{Z}) \geq \operatorname{rank}_{\mathbb{Z} / 2 \mathbb{Z}} \widehat{H F}(\Sigma(L) ; \mathbb{Z} / 2 \mathbb{Z}) .
$$

The left-hand side of this inequality is equal to $\operatorname{rank}_{\mathbb{Z} / 2 \mathbb{Z}} \widetilde{K h}(L ; \mathbb{Z} / 2 \mathbb{Z})$ by a duality theorem for Khovanov homology under taking mirror images [16, Corollary 11]. We have

$$
\operatorname{rank}_{\mathbb{Z} / 2 \mathbb{Z}} \widehat{H F}(\Sigma(L) ; \mathbb{Z} / 2 \mathbb{Z}) \geq \operatorname{rank}_{\mathbb{Z}} \widehat{H F}(\Sigma(L)),
$$

by the universal coefficients theorem. Multiplying by 2 and combining with Equation (1) yields the desired inequality. 


\section{Manifolds with rank 2 Heegaard Floer homology}

In this section we prove our theorem characterizing manifolds with rank 2 Floer homology. The basic idea of the proof is simple. We first use the rank assumption to show that the only nontrivial class in $H_{2}(Y ; \mathbb{Z})$ is represented by a torus. The rank being this small further implies, by work of $\mathrm{Ai}$ and the second author [1], that this torus is a fiber in a fibration of the three-manifold over the circle. The scarcity of three-manifolds which fiber in this way then pins down the manifold exactly.

Proof of Theorem 1.1 Suppose $Y$ is a closed irreducible 3-manifold with $b_{1}(Y)>0$ and $\operatorname{rank} \widehat{H F}(Y)=2$.

Claim $1 Y$ contains a nonseparating torus.

Since $b_{1}(Y)>0$, there exists a closed connected surface $F \subset Y$, such that $[F] \neq$ $0 \in H_{2}(M)$ and $F$ is Thurston norm minimizing. $F$ is not a sphere since $Y$ is irreducible so, in particular, $F$ is taut. By Theorem 2.2, $\widehat{H F}(Y,[F], g-1) \neq 0$, where $g$ is the genus of $F$. A symmetry property of Floer homology further implies that $\widehat{H F}(Y,[F], 1-g) \neq 0\left[28\right.$, Theorem 2.4]. Note that since $b_{1}(Y)>0$, [28, Proposition 5.1] implies $\chi(\widehat{H F}(Y, \mathfrak{s}))=0$. In particular $\widehat{H F}(Y,[F], g-1)$ and $\widehat{H F}(Y,[F], 1-g)$ each have rank at least 2 . If $g>1$, then

$$
\operatorname{rank} \widehat{H F}(Y) \geq \operatorname{rank} \widehat{H F}(Y,[F], g-1)+\operatorname{rank} \widehat{H F}(Y,[F], 1-g) \geq 4,
$$

which is impossible. So $F$ must be a torus.

Claim $2 \quad Y$ is a torus bundle over the circle.

The tool for proving this claim will be the main theorem of [1], which indicates that Floer homology detects torus fibrations:

Theorem 3.1 [1, Theorem 1.2] Let $Y$ be an irreducible oriented 3-manifold. Suppose $F \subset Y$ is an embedded torus and there exists $\omega \in H_{1}(Y ; \mathbb{Z})$ satisfying $\omega \cdot[F] \neq 0$, and for which

$$
\operatorname{rank}_{\Lambda} \underline{H F}^{+}\left(Y ; \Lambda_{\omega}\right)=1 .
$$

Then $Y$ fibers over the circle with fiber $F$.

Note that to be consistent, we have stated this theorem in terms of coefficients twisted by $\omega \in H_{1}(Y ; \mathbb{Z})$ rather than $\omega \in H^{2}(Y ; \mathbb{R})$ as in [1]. The definition of the modules 
involved, together with Poincaré duality, shows that the chain complexes are isomorphic. Also recall that $\widehat{H F}\left(Y ; \Lambda_{\omega}\right)$ denotes the sum of Floer groups over all $\operatorname{Spin}^{c}$ structures.

We wish to apply the above theorem. By Theorem 2.1, we can choose a homology class $\omega \in H_{1}(Y)$ with $\omega \cdot[F] \neq 0$ and $\underline{H F}+\left(Y,[F], 0 ; \Lambda_{\omega}\right) \neq 0$. Moreover, an application of the adjunction inequality [28, Theorem 7.1] shows that $\underline{H F}^{+}\left(Y,[F], 0 ; \Lambda_{\omega}\right)=$ $\mathrm{HF}^{+}\left(Y ; \Lambda_{\omega}\right)$. More precisely, the adjunction inequality adapted to twisted coefficients tells us that $\operatorname{HF}^{+}\left(Y, \mathfrak{s} ; \Lambda_{\omega}\right)=0$ for any $\operatorname{Spin}^{c}$ structure satisfying $\left\langle c_{1}(\mathfrak{s}),[F]\right\rangle \neq$ 0 . Thus it remains to show that our assumption $\operatorname{rank}_{\mathbb{Z}} \widehat{H F}(Y)=2$ implies that $\operatorname{rank}_{\Lambda} \underline{H F}^{+}\left(Y,[F], 0 ; \Lambda_{\omega}\right)=1$. This will follow easily from the universal coefficients theorem and the exact sequence relating $\underline{H F^{+}}$to $\underline{\widehat{H F}}$.

As an intermediary, let us consider the $\omega$-twisted Heegaard Floer homology

$$
\widehat{\widehat{H F}}\left(Y,[F], 0 ; R_{\omega}\right)
$$

(recall that $R=\mathbb{Q}\left[T, T^{-1}\right]$ ). As in Section 2.1 we have two natural $R$-modules: the trivial module $\mathbb{Q}$ and the universal Novikov ring $\Lambda$. Correspondingly, we have the untwisted Heegaard Floer homology $\widehat{H F}(Y,[F], 0 ; \mathbb{Q})$ and the $\omega$-perturbed Floer homology $\widehat{H F}\left(Y,[F], 0 ; \Lambda_{\omega}\right)$.

We can apply the universal coefficients theorem to get

$$
\widehat{H F}(Y,[F], 0 ; \mathbb{Q}) \cong\left(\underline{\widehat{H F}}\left(Y,[F], 0 ; R_{\omega}\right) \otimes_{R} \mathbb{Q}\right) \oplus \operatorname{Tor}_{R}\left(\underline{\widehat{H F}}\left(Y,[F], 0 ; R_{\omega}\right), \mathbb{Q}\right) .
$$

By assumption, we have $\widehat{H F}(Y,[F], 0 ; \mathbb{Q}) \cong \mathbb{Q}^{2}$, so

$$
\operatorname{rank}_{R} \underline{\widehat{H F}}\left(Y,[F], 0 ; R_{\omega}\right) \leq 2 .
$$

Again by the universal coefficients theorem, we have

$$
\underline{\widehat{H F}}\left(Y,[F], 0 ; \Lambda_{\omega}\right) \cong\left(\underline{\widehat{H F}}\left(Y,[F], 0 ; R_{\omega}\right) \otimes_{R} \Lambda\right) \oplus \operatorname{Tor}_{R}\left(\underline{\widehat{H F}}\left(Y,[F], 0 ; R_{\omega}\right), \Lambda\right) .
$$

Since $\Lambda$ is $R$-torsion free, we have $\operatorname{Tor}_{R}\left(\widehat{\hat{H F}}\left(Y,[F], 0 ; R_{\omega}\right), \Lambda\right)=0$. Thus

$$
\operatorname{rank}_{\Lambda} \underline{\widehat{H F}}\left(Y,[F], 0 ; \Lambda_{\omega}\right) \leq 2 .
$$

The proof of the following lemma will be given in the Appendix.

Lemma 3.2 Suppose $Y$ contains a torus $F$ and $\omega \in H_{1}(Y)$ satisfies $\omega \cdot[F] \neq 0$. Then the $U$-action

$$
U: \underline{H F}^{+}\left(Y ; \Lambda_{\omega}\right) \rightarrow \underline{H F}^{+}\left(Y ; \Lambda_{\omega}\right)
$$

is zero. 
Having bounded the rank of $\underline{\widehat{H F}}$, recall the exact sequence

$$
\cdots \rightarrow \underline{H F}^{+} \stackrel{U}{\longrightarrow} \underline{H F^{+}} \rightarrow \underline{\widehat{H F}} \rightarrow \cdots .
$$

By Lemma 3.2, $U=0$. Since $\underline{H F}+\left(Y,[F], 0 ; \Lambda_{\omega}\right) \neq 0$, we must have

$$
\operatorname{rank}_{\Lambda} \underline{H F}^{+}\left(Y,[F], 0 ; \Lambda_{\omega}\right)=1,
$$

which completes the proof of the claim.

Claim $3 \quad Y$ is obtained by zero surgery on the trefoil knot.

Since $Y$ is a torus bundle, $b_{1}(Y) \leq 3$. If $b_{1}(Y)=3$, then $Y=T^{3}$, for which $\widehat{H F}$ is known to be isomorphic to $\mathbb{Z}^{6}$ [25, Proposition 8.4]. If $b_{1}(Y)=2$ or $H_{1}(Y ; \mathbb{Z})$ contains torsion, [28, Theorem 10.1] and Lemma 2.3 imply that $\operatorname{rank} \widehat{H F}(Y) \geq 4$. Thus $H_{1}(Y ; \mathbb{Z}) \cong \mathbb{Z}$.

Let $A \in S L(2, \mathbb{Z})$ be the matrix representing the monodromy of the torus bundle. In order to have $H_{1}(Y ; \mathbb{Z}) \cong \mathbb{Z}$, we must have $\operatorname{det}(A-l)= \pm 1$ (by Mayer-Vietoris), so trace $A=1$ or 3 . Up to conjugacy in $S L(2, \mathbb{Z})$, there are only three such matrices $[41,21.15]$, which correspond to the zero surgeries on the two trefoil knots and on the figure 8 knot. The first two manifolds have $\widehat{H F} \cong \mathbb{Z}^{2}$ while the last one has $\widehat{H F} \cong \mathbb{Z}^{4}$ [25, Section 8].

\section{The zero surgery on the trefoil as a branched double cover}

For a link $L \subset S^{3}$, we let $\Sigma(L)$ denote the branched double cover of $S^{3}$, branched along $L$. We will denote the manifold obtained by zero surgery on the trefoil by $M$. In this section, we classify the links for which $\Sigma(L) \cong M$. It turns out there are only two.

Proposition 4.1 Suppose $\Sigma(L) \cong M$, the manifold obtained by 0 -surgery on the trefoil knot. Then $L$ is isotopic to one of the two links, $H_{1,3}$ or $H_{-1,-3}$, pictured in Figure 1.

Remark We consider $M$ as an unoriented manifold, and hence the links are specified up to taking mirror images.

We will prove the proposition by a geometric argument, which we now sketch. The argument begins with the observation that $M$ admits a Euclidean geometric structure. Existing results about group actions on such manifolds will show that the involution 
presenting $M$ as a branched double cover can be assumed to be an isometry. Analyzing this situation, we will show that the involution restricts to an involution on the two pieces of the decomposition of $M$ as a torus bundle

$$
M \cong T^{2} \times I \cup_{A} T^{2} \times I .
$$

Using this fact, we can show that $L$ must result from 2-cabling each component of the Hopf link, as shown in Figure 1. An analysis of $H_{1}(\Sigma(L) ; \mathbb{Z})$ for such $L$ then specifies the link exactly. With the general idea in place, we begin.

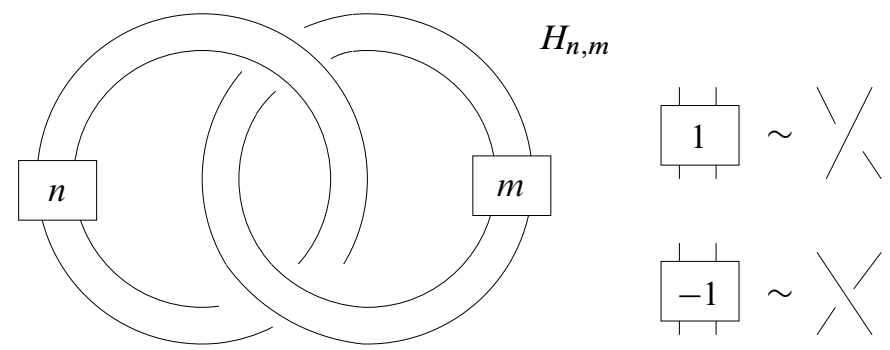

Figure 1: A satellite of the Hopf link formed by 2-cabling each of its components. The numbers $n$ and $m$ indicate the number of crossings, according to the convention on the right.

Proof of Proposition 4.1 Suppose $M$ is homeomorphic to $\Sigma(L)$. Let $\pi: M \rightarrow S^{3}$ be the branched covering map, and let $\rho: M \rightarrow M$ be the deck transformation, which is an involution. Then $\pi^{-1}(L)$ is the set of fixed points of $\rho$. The following lemma captures the geometry of our setup.

Lemma 4.2 There exists a flat metric on $M$ which is preserved by $\rho$. With respect to this metric, there exists a totally geodesic (embedded) torus $T$ representing a generator of $H_{2}(M) \cong \mathbb{Z}$.

Proof $M$ is a torus bundle over $S^{1}$ with a 6 -periodic monodromy, so the 6 -fold cyclic cover of $M$ is $T^{3}$. Thus $M$ admits a Euclidean structure. According to [20, Theorem 2.1], there exists a flat metric on $M$ with respect to which $\rho$ is an isometry.

Suppose $f: T^{2} \rightarrow M$ is a least area map representing a generator of $H_{2}(M)$. [7, Theorem 5.1] implies that either (i) $f$ is an embedding, or (ii) $f$ double covers an embedded one-sided surface in $M$. The latter is ruled out by the fact that $f_{*}\left(\left[T^{2}\right]\right) \neq$ $0 \in H_{2}(M)$, hence $f$ is an embedding (strictly speaking, to apply the theorem we must also verify that $M$ is irreducible and contains no $\mathbb{R} P^{2}$, but this follows easily from the fact that $M$ is a torus bundle). Let $T$ denote the image of $f$. Since $T$ 
is area minimizing, it is a minimal surface, and hence the mean curvature vector vanishes identically. Since $M$ is flat, the Gaussian curvature of $T$ is the product of its principal curvatures, and is nonpositive since the mean curvature (the average of the principal curvatures) vanishes. The Gauss-Bonnet Theorem then implies that the Gaussian curvature of $T$ is 0 everywhere, and hence the principal curvatures also vanish everywhere, so $T$ is totally geodesic.

The geometry at hand tightly constrains the behavior of the covering involution with regard to the torus. We have the following

Lemma 4.3 In the previous lemma, one can assume that the totally geodesic torus satisfies $\rho(T) \cap T=\varnothing$ and $[\rho(T)]=-[T] \in H_{2}(M)$.

Proof Since $H_{2}(M) \cong \mathbb{Z}, \rho(T)$ is homologous to $\pm T$. Endow the three-torus, $T^{3}$, with the flat metric obtained by pulling back the metric on $M$ under the 6 -fold cyclic covering map $p: T^{3} \rightarrow M$. We will compare the lifts of $T$ and $\rho(T)$ under $p$.

Let $\widetilde{T}$ denote a component of $p^{-1}(T) . \widetilde{T}$ is, like $T$, totally geodesic. The surface $p^{-1} \circ \rho(T)$ is a disjoint union of totally geodesic tori in $T^{3}$, such that each component is homologous to $\pm[\widetilde{T}]$. We claim that $\widetilde{T}$ is either a component of $p^{-1} \circ \rho(T)$ or disjoint from $p^{-1} \circ \rho(T)$. This follows from elementary Euclidean geometry. Indeed, since all the tori are geodesic they lift to planes in the universal cover, $\mathbb{E}^{3}$. The assumption that the tori are homologous up to sign implies that these planes are parallel or have a pair of components which coincide (if not, they would intersect in a line that projects to a nonseparating circle of intersection between the tori in $T^{3}$, contradicting the homological assumption). This proves the claim. In the case that $\widetilde{T}$ is disjoint from $p^{-1} \circ \rho(T)$ we see that $\rho(T) \cap T=\varnothing$, as desired. If $\widetilde{T}$ is a component of $p^{-1} \circ \rho(T)$, then $\rho(T)=T$ as a set.

In the case that $\rho(T)=T$, we claim that $\rho_{*}[T]=-[T]$. Suppose, otherwise, that $\rho_{*}[T]=[T]$. Then $\left.\rho\right|_{T}$ is an orientation-preserving involution. Thus $\pi(T)$ is a closed oriented surface in $S^{3}$. Choose a simple closed curve $\gamma \subset M$ intersecting $T$ exactly once. Then $\pi(\gamma)$ is a closed curve in $S^{3}$ which has algebraic intersection number 2 with $\pi(T)$, contradicting the fact that $H_{1}\left(S^{3}\right)=0$.

Thus $\rho_{*}[T]=-[T]$ if $\rho(T)=T$. For some small $\varepsilon>0$, consider the set

$$
N_{\varepsilon}=\{x \in M \mid \operatorname{dist}(x, T) \leq \varepsilon\}
$$

Then $\partial N_{\varepsilon}$ consists of two flat tori, $T_{\varepsilon}$ and $T_{-\varepsilon}$. The assumptions that $\rho_{*}[T]=-[T]$ and $\rho(T)=T$ imply that $\rho\left(T_{\varepsilon}\right)=T_{-\varepsilon}$. By working with $T_{\varepsilon}$ instead of $T$, we obtain the desired conclusion. 
Finally, if $\rho(T) \cap T=\varnothing$, we claim that $\rho_{*}[T]=-[T]$ also holds. Indeed, $T$ and $\rho(T)$ split $M$ into two parts, $C_{1}$ and $C_{2}$, both of which are homeomorphic to $T^{2} \times I$. Now if $\rho_{*}[T]=[T]$, then $\rho$ would have to switch $C_{1}$ and $C_{2}$. Since $\rho$ has no fixed points on $\partial C_{1}=T \cup \rho(T)$, this would imply that $\rho$ is free, a contradiction.

Next, we show that $L$ must result from 2-cabling the components of the Hopf link.

Lemma 4.4 If $M=\Sigma(L)$, then there exists a genus one Heegaard splitting $S^{3}=$ $V_{1} \cup V_{2}$, such that $L$ is the union of a closed 2-braid in $V_{1}$ and a closed 2-braid in $V_{2}$ (see Figure 1).

Proof The last lemma showed that we can assume $T$ and $\rho(T)$ split $M$ into two parts $C_{1}$ and $C_{2}$, with each $C_{i}$ homeomorphic to $T^{2} \times I$. Moreover, since $\rho_{*}[T]=-[T]$, we have $\rho\left(C_{i}\right)=C_{i}$, and $V_{i}=\pi\left(C_{i}\right)$ is a manifold with torus boundary. Since $\pi(T)$ is an embedded torus in $S^{3}$, the two manifolds $V_{1}, V_{2}$ bounded by $\pi(T)$ have the same homology groups as the solid torus.

Let $h$ be a generator of $H_{2}\left(V_{1}, \partial V_{1}\right) \cong \mathbb{Z}$. Since $\pi: C_{1} \rightarrow V_{1}$ is a proper map of nonzero degree, there exists a primitive element $\tilde{h} \in H_{2}\left(C_{1}, \partial C_{1}\right)$ such that $\pi_{*}(\tilde{h})=2 h$. We can choose an annulus $A \subset C_{1}=T^{2} \times I$ representing $\widetilde{h}$, hence $\pi(A)$ represents $2 h$. By Gabai's theorem that the singular Thurston norm is equal to the Thurston norm $[8$, Corollary 6.18], the Thurston norm of $2 h$ is 0 , hence the Thurston norm of $h$ is also 0 . Let $G \subset V_{1}$ be a Thurston norm minimizing surface in the homology class $h$. Then $\partial G$ represents a primitive element in $H_{1}\left(\partial V_{1}\right)$. Attaching annuli to $\partial G$ if necessary, we may assume $|\partial G|=1$, and hence the component of $G$ containing $\partial G$ is a disk. Since $V_{1} \subset S^{3}$, it follows that $V_{1}$ is a solid torus.

Now $C_{1}$ is homeomorphic to $T^{2} \times[0,1]$, and $\partial C_{1}$ consists of two parallel flat tori. The universal cover $\widetilde{C_{1}}$ of $C_{1}$ is a submanifold of $\mathbb{E}^{3}$ bounded by two parallel planes. After scaling the metric, $\widetilde{C_{1}}$ is isometric to $\mathbb{E}^{2} \times[0,1]$ and each $\mathbb{E}^{2} \times t$ is preserved by the (isometric) action of $\pi_{1}\left(C_{1}\right)$. Let $R_{t}=\left(\mathbb{E}^{2} \times t\right) / \pi_{1}\left(C_{1}\right)$. Then $C_{1}$ is foliated by the flat tori $R_{t}$, and the distance between $R_{t}$ and $R_{0}$ is $t$. Since $\rho$ is an isometry and $\rho\left(R_{0}\right)=R_{1}, \rho$ must send $R_{t}$ to $R_{1-t}$.

Now as the fixed point set of an isometry, $\pi^{-1}(L)$ is geodesic. Each $C_{i}$ must contain some components of $\pi^{-1}(L)$. Let $K=\pi^{-1}(L) \cap C_{1}$. Since $K$ is a geodesic disjoint from the flat tori $R_{0}$ and $R_{1}, K$ should be parallel to them. Indeed, since $\rho$ is an isometry, for each component $K_{i}$ of $K$

$$
\operatorname{dist}\left(K_{i}, R_{0}\right)=\operatorname{dist}\left(\rho\left(K_{i}\right), \rho\left(R_{0}\right)\right)=\operatorname{dist}\left(K_{i}, R_{1}\right),
$$


so $K_{i}$ lies on the torus $R_{1 / 2}$. Hence $K \subset R_{1 / 2}$. Let $R_{[0,1 / 2]}=\bigcup_{t \in[0,1 / 2]} R_{t}$, then $R_{[0,1 / 2]}$ is homeomorphic to $T^{2} \times[0,1 / 2]$, and $\rho\left(R_{[0,1 / 2]}\right) \cap R_{[0,1 / 2]}=R_{1 / 2}$. Choose a properly embedded surface $W \subset R_{[0,1 / 2]}$ such that each component of $W$ is an annulus whose boundary consists of a component of $K$ and a curve on $R_{0}$. Then $\pi(W)$ is a disjoint union of annuli in $V_{1}=\pi\left(C_{1}\right)$, where each annulus connects a component of $L$ to an essential curve on $\partial V_{1}$. It follows that $L \cap V_{1}$ is a torus link in the solid torus $V_{1}$. A simple Euler characteristic count shows that $L$ intersects each meridian disk of $V_{1}$ in two points, so $L \cap V_{1}$ is a 2-braid in $V_{1}$.

The same argument as above shows that $V_{2}$ is a solid torus, and $L \cap V_{2}$ is a 2-braid in $V_{2}$. Now $V_{1} \cup V_{2}$ is a genus one Heegaard splitting for $S^{3}$.

To complete the theorem, let $L=H_{m, n} \subset S^{3}$ be the link from the previous lemma, such that $H_{m, n} \cap V_{1}$ is isotopic to the $(2, m)$ torus link in $V_{1}$ and $H_{m, n} \cap V_{2}$ is isotopic to the $(2, n)$ torus link in $V_{2}$.

Lemma 4.5 The manifold $\Sigma\left(H_{m, n}\right)$ is a torus bundle, and the monodromy is represented by the matrix

$$
\left(\begin{array}{cc}
m n-1 & n \\
-m & -1
\end{array}\right)
$$

Proof The proof of Lemma 4.4 shows that $\Sigma\left(H_{m, n}\right)$ is a torus bundle. We only need to determine its monodromy.

Choose two curves $\mu, \lambda$ on the surface $\partial V_{1}=-\partial V_{2}$, such that $\mu, \lambda$ are the meridians of $V_{1}, V_{2}$, respectively. Moreover, the curves are oriented such that $\mu \cdot \lambda=1$.

Let $T_{1}, T_{2}$ be the two components of $\pi^{-1}\left(\partial V_{1}\right)$. Let

$$
\tilde{\mu}_{i}=\pi^{-1}(\mu) \cap T_{i}, \quad \tilde{\lambda}_{i}=\pi^{-1}(\lambda) \cap T_{i} .
$$

The preimage of the meridian disk of $V_{1}$ is an annulus which gives a homology (in $\left.C_{1}=\pi^{-1}\left(V_{1}\right)\right)$ between $\left[\tilde{\mu}_{1}\right]$ and $-\left[\tilde{\mu}_{2}\right]$, so

$$
\left[\tilde{\mu}_{1}\right]=-\left[\tilde{\mu}_{2}\right] \in H_{1}\left(C_{1}\right) .
$$

Moreover, there is a compact surface $A \subset V_{1}$, such that $A$ is an annulus when $m$ is odd and $A$ is the union of two annuli when $m$ is even, and $\partial A$ consists of $H_{m, n} \cap V_{1}$ and a $(2, m)$ torus link on $\partial V_{1}$. The preimage of $A$ gives a homology (in $\left.C_{1}\right)$ between $2\left[\tilde{\lambda}_{1}\right]+m\left[\tilde{\mu}_{1}\right]$ and $2\left[\tilde{\lambda}_{2}\right]+m\left[\tilde{\mu}_{2}\right]$. Since $\left[\tilde{\mu}_{1}\right]=-\left[\tilde{\mu}_{2}\right]$ in $C_{1}$, it follows that

$$
\left[\tilde{\lambda}_{1}\right]=\left[\tilde{\lambda}_{2}\right]+m\left[\tilde{\mu}_{2}\right] \in H_{1}\left(C_{1}\right) .
$$


In $V_{2}$, the roles of $\lambda$ and $\mu$ are switched. The same argument as above shows that

$$
\left[\tilde{\lambda}_{1}\right]=-\left[\tilde{\lambda}_{2}\right], \quad\left[\tilde{\mu}_{1}\right]=\left[\tilde{\mu}_{2}\right]+n\left[\tilde{\lambda}_{2}\right]
$$

in $H_{1}\left(C_{2}\right)$.

Consider the manifold $C_{1} \cup_{T_{2}} C_{2}$, which is homeomorphic to $T^{2} \times I$. There are two copies of $\tilde{\mu}_{1}, \tilde{\lambda}_{1}$ on its boundary, and their homological relation can be computed as follows:

$$
\begin{aligned}
{\left[\tilde{\mu}_{1}\right] } & =\left[\tilde{\mu}_{2}\right]+n\left[\tilde{\lambda}_{2}\right] \\
& =-\left[\tilde{\mu}_{1}\right]+n\left(\left[\tilde{\lambda}_{1}\right]+m\left[\tilde{\mu}_{1}\right]\right) \\
& =(m n-1)\left[\tilde{\mu}_{1}\right]+n\left[\tilde{\lambda}_{1}\right] \\
{\left[\tilde{\lambda}_{1}\right] } & =-\left[\tilde{\lambda}_{2}\right] \\
& =-\left(\left[\tilde{\lambda}_{1}\right]+m\left[\tilde{\mu}_{1}\right]\right) .
\end{aligned}
$$

So the monodromy of the torus bundle is given by the matrix

$$
\left(\begin{array}{cc}
m n-1 & n \\
-m & -1
\end{array}\right)
$$

We are now able to finish the proof of Proposition 4.1. If $\Sigma\left(H_{m, n}\right)$ is homeomorphic to the zero surgery on the trefoil knot, then the monodromy of the torus bundle has order 6 . This implies that the trace of the matrix is 1 . By the preceding lemma we find that $m n=3$, and hence $m= \pm 1, n= \pm 3$ or $m= \pm 3, n= \pm 1$. Since $H_{n, m} \simeq H_{m, n}$, the link is $H_{1,3}$ or $H_{-1,-3}$.

Remark Note that our proof applies equally well to classify links whose branched double cover gives rise to any torus bundle possessing a Euclidean structure. In particular, we recover the well-known result that $T^{3}$ is not a branched double cover of a link in $S^{3}$ [14].

\section{Links with rank 4 Khovanov homology}

In this section, we prove Theorem 1.3. The theorem will follow quickly from the results of the preceding two sections, together with Proposition 2.6. It will be useful, however, to first understand essential spheres in branched double covers. The following proposition is a well-known consequence of the Equivariant Sphere Theorem, originally proved by Meeks, Scott and Yau [21]. (See also Dunwoody [4] for the version we use here.) 
Proposition 5.1 (1) Suppose $L$ is a link in $S^{3}$. If $L=L_{1} \# L_{2}$, then $\Sigma(L)=$ $\Sigma\left(L_{1}\right) \# \Sigma\left(L_{2}\right)$. If $L=L_{1} \sqcup L_{2}$, then $\Sigma(L)=S^{1} \times S^{2} \# \Sigma\left(L_{1}\right) \# \Sigma\left(L_{2}\right)$.

(2) Suppose $L$ is a nonsplit prime link in $S^{3}$, then $\Sigma(L)$ is irreducible.

(3) If $L$ is a nonsplit link, then $\Sigma(L)$ contains no $S^{1} \times S^{2}$ summand.

\section{Proof (1) This fact is obvious.}

(2) Assume $\Sigma(L)$ is reducible. By the Equivariant Sphere Theorem, there exists an essential sphere $S \subset \Sigma(L)$, such that $\rho(S)=S$ or $\rho(S) \cap S=\varnothing$.

If $\rho(S)=S$, then $S$ doubly branched covers $\pi(S)$, hence $\pi(S)$ is an embedded surface in $S^{3}$, and it is either a disk, a sphere or a projective plane. The last case is immediately ruled out since $S^{3}$ does not contain any embedded projective plane.

If $\pi(S)$ is a disk, then $\partial(\pi(S))$ is a component of $L$ which bounds a disk in the complement of $L$. This contradicts the assumption that $L$ is nonsplit.

If $\pi(S)$ is a sphere, then $\left.\pi\right|_{S}$ is a 2 -fold branched covering with two ramification points. So $L$ intersects $\pi(S)$ in exactly two points. The sphere $\pi(S)$ splits $S^{3}$ into two balls $B_{1}, B_{2}$. If $L \cap B_{i}$ is a trivial arc in $B_{i}$, then $\pi^{-1}\left(B_{i}\right)$ is a ball bounded by $S$ in $\Sigma(L)$, contradicting the assumption that $S$ is essential. So $L$ is a nontrivial connected sum, which is impossible since $L$ is prime.

If $\rho(S) \cap S=\varnothing$, then $\pi(S)$ is an embedded sphere in the complement of $L$. Since $L$ is nonsplit, $\pi(S)$ bounds a ball in $S^{3}-L$. It follows that $S$ bounds a ball in $\Sigma(L)$, a contradiction.

(3) If $L$ is nonsplit, there exists a collection of spheres $S_{1}, \ldots, S_{n} \subset S^{3}$, such that each $S_{i}$ intersects $L$ in exactly two points, and they decompose $L$ as a connected sum of nonsplit prime links $L_{1}, \ldots, L_{n+1}$. Thus $\Sigma(L)$ is a connected sum of $\Sigma\left(L_{i}\right)$ 's. By (2), each $\Sigma\left(L_{i}\right)$ is irreducible, so $\Sigma(L)$ contains no $S^{1} \times S^{2}$ summand.

Proof of Theorem 1.3 Let $L$ be a link with $\operatorname{det}(L)=0$. By Theorem 2.4, if $L$ is an $n$-component link, then $\operatorname{rank} K h(L) \geq 2^{n}$. So we can assume $L$ has two components (it cannot have one-component, since knots satisfy $\operatorname{det}(K) \neq 0$ ).

If $L$ is split with two components $K_{1}, K_{2}$, then

$$
\operatorname{rank}_{\mathbb{F}} K h(L ; \mathbb{F})=\operatorname{rank}_{\mathbb{F}} K h\left(K_{1} ; \mathbb{F}\right) \times \operatorname{rank}_{\mathbb{F}} K h\left(K_{2} ; \mathbb{F}\right),
$$

where $\mathbb{F}=\mathbb{Z} / 2 \mathbb{Z}$. Indeed, there are chain complexes for which $C K h(L) \cong C K h\left(K_{1}\right) \otimes$ $C K h\left(K_{2}\right)$. Thus $L$ has Khovanov rank 4 (over $\mathbb{F}$ ) if and only if each $K_{i}$ has Khovanov rank 2. 
If $L$ is nonsplit, then $\Sigma(L)$ contains no $S^{1} \times S^{2}$ summand by Proposition 5.1. Since $\operatorname{det}(L)=0, b_{1}(\Sigma(L))>0$ (recall that $|\operatorname{det}(L)|=\left|H_{1}(\Sigma(L) ; \mathbb{Z})\right|$ when $\operatorname{det}(L) \neq 0$ and, if $\operatorname{det}(L)=0$, that $H_{1}(\Sigma(L) ; \mathbb{Z})$ has infinite order). Moreover, the fact that $L$ has two components implies that $b_{1}(\Sigma(L)) \leq 1$, and hence $b_{1}(\Sigma(L))=1$.

Since $\operatorname{rank}_{\mathbb{F}} K h(L ; \mathbb{F})=4$, Proposition 2.6 implies that $\operatorname{rank} \widehat{H F}(\Sigma(L)) \leq 2$. For a manifold with $b_{1}(M)=1$, [28, Theorem 10.1] and Lemma 2.3 imply that $\operatorname{rank} \widehat{H F}(M) \geq 2$, so we see that $\operatorname{rank} \widehat{H F}(\Sigma(L))=2$. Calling on Corollary 1.2, we see that $\Sigma(L)=$ $\pm S_{0}^{3}\left(3_{1}\right) \# Z$ for some homology sphere, $Z$. Now by Proposition 5.1 and the uniqueness of the Kneser-Milnor prime decomposition, $L$ has a connected summand, $L_{0}$, such that $\Sigma\left(L_{0}\right)= \pm S_{0}^{3}\left(3_{1}\right)$. Proposition 4.1 shows that, up to taking mirror images, $L_{0}$ is either $H_{-1,-3}$ or $H_{1,3}$. Since $L_{0}$ has two components, the other prime summands of $L$ are all knots.

For the reduced Khovanov homology, we have the connected sum formula [17]

$$
\widetilde{K h}\left(L^{\prime} \# L^{\prime \prime}\right) \cong \widetilde{K h}\left(L^{\prime}\right) \otimes \widetilde{K h}\left(L^{\prime \prime}\right),
$$

which implies that the rank of $\widetilde{K h}(L)$ is not less than the rank of $\widetilde{K h}\left(L_{0}\right)$.

If $L_{0}=H_{-1,-3}$, the Jones polynomial of $H_{-1,-3}$ is

$$
-t^{-23 / 2}+t^{-21 / 2}-t^{-13 / 2}-t^{-9 / 2}
$$

so the rank of $\widetilde{K h}(L)$ is at least 4 . The same rank estimate works when $L_{0}=H_{1,3}$. It follows from (1) that the rank of $K h(L)$ is at least 8 , a contradiction. This implies that $L$ must be split.

Proof of Corollary 1.4 Assume that Khovanov homology detects the unknot. If $K h(L) \cong K h\left(U_{2}\right)$, where $U_{2}$ is the two-component unlink, then $\operatorname{rank} K h(L)=4$. Also, the Jones polynomial, $J_{L}(q)$, is equal to that of the unlink. In particular, we have $|\operatorname{det}(L)|=\left|J_{L}(-1)\right|=0$. Theorem 1.3 now shows that $L$ is isotopic to a split link, each component of which has the Khovanov homology of the unknot. Our assumption implies that both components are unknotted.

Conversely, if Khovanov homology detects the two-component unlink, then it clearly detects the unknot (given a knot $K$, consider the split link obtained from $K$ union an unknot).

\section{Appendix: The proof of Lemma 3.2}

In this appendix, we give a proof of Lemma 3.2. We first learned this result from Peter Ozsváth. The argument presented here was told to us by Yanki Lekili. 
Suppose $W$ is a cobordism from $Y_{1}$ to $Y_{2}, \Omega \in H_{2}(W, \partial W)$, and $\omega_{1}, \omega_{2}$ are the restriction of $\Omega$ on $Y_{1}, Y_{2}$, respectively (ie the image of $\Omega$ under the natural map $\left.H_{2}(W, \partial W) \rightarrow H_{1}(\partial W)\right)$. Then there is a map [33, Section 2.7]

$$
\underline{F_{W, \Omega}^{+}}: \underline{H F}^{+}\left(Y_{1} ; \Lambda_{\omega_{1}}\right) \rightarrow \underline{H F}^{+}\left(Y_{2} ; \Lambda_{\omega_{2}}\right) .
$$

Consider the cobordism $W=Y \times I$. Let $\gamma$ be an arc connecting $Y \times 0$ to $F \times 1 / 2$. Let $W_{1}$ be the neighborhood of $(Y \times 0) \cup \gamma \cup(F \times 1 / 2)$ in $W$, and let $W_{2}=\overline{W-W_{1}}$. Then $W_{1}$ is a cobordism from $Y$ to $Y^{\prime}=Y \#\left(F \times S^{1}\right)$. Given $\omega \in H_{1}(Y)$, let $\Omega=\omega \times[I] \in H_{2}(W, \partial W)$, and let $\Omega_{i}$ be the restriction of $\Omega$ to $H_{2}\left(W_{i}, \partial W_{i}\right)$. Considering the maps on $\underline{H F}^{+}\left(-; \Lambda_{\omega}\right)$ induced by cobordisms, we have

$$
\mathrm{Id}=\underline{F_{W, \Omega}^{+}}=\underline{F_{W_{2}, \Omega_{2}}^{+}} \circ \underline{F_{W_{1}, \Omega_{1}}^{+}}: \underline{H F}^{+}\left(Y ; \Lambda_{\omega}\right) \rightarrow \underline{H F}^{+}\left(Y ; \Lambda_{\omega}\right),
$$

which factors through $\underline{H F^{+}}\left(Y^{\prime} ; \Lambda_{\Omega \mid Y^{\prime}}\right)$. Here $\Omega \mid Y^{\prime}$ is a homology class in $H_{1}\left(Y^{\prime}\right)$ which is the intersection of $\Omega$ and $\left[Y^{\prime}\right]$. We claim that $\left(\Omega \mid Y^{\prime}\right) \cdot[F] \neq 0$. In fact, let $\Omega^{*} \in H^{2}(W)$ be the Poincaré dual of $\Omega$, and let $e: Y^{\prime} \rightarrow W$ be the inclusion map. Then $\operatorname{PD}^{-1}\left(\Omega \mid Y^{\prime}\right)=e^{*}\left(\Omega^{*}\right)$. It follows that

$$
\begin{aligned}
\left(\Omega \mid Y^{\prime}\right) \cdot[F] & =\left\langle e^{*}\left(\Omega^{*}\right),[F]\right\rangle \\
& =\left\langle\Omega^{*}, e_{*}[F]\right\rangle \\
& =\Omega \cdot[F] \\
& \neq 0 .
\end{aligned}
$$

Since $H_{1}\left(Y^{\prime}\right) \cong H_{1}(Y) \oplus H_{1}\left(F \times S^{1}\right)$, we can further restrict $\Omega \mid\left(F \times S^{1}\right)$ and, according to [2, Theorem 1.1] and the claim, we have

$$
\underline{H F}^{+}\left(F \times S^{1} ; \Lambda_{\Omega \mid\left(F \times S^{1}\right)}\right) \cong \Lambda .
$$

Adapted to twisted coefficients, the product theorem for the Floer homology of connected sums [28, Theorem 6.2] yields isomorphisms

$$
\begin{aligned}
\underline{H F}^{-}\left(Y^{\prime} ; \Lambda_{\Omega \mid Y^{\prime}}\right) \cong H_{*}\left(\underline{C F}^{-}\left(Y ; \Lambda_{\Omega \mid Y}\right) \otimes_{\Lambda[U]} \underline{C F}^{-}\left(F \times S^{1} ; \Lambda_{\Omega \mid\left(F \times S^{1}\right)}\right)\right), \\
\underline{H F}^{\infty}\left(Y^{\prime} ; \Lambda_{\Omega \mid Y^{\prime}}\right) \cong H_{*}\left(\underline{C F}^{\infty}\left(Y ; \Lambda_{\Omega \mid Y}\right) \otimes_{\Lambda\left[U, U^{-1}\right]} \underline{C F^{\infty}}\left(F \times S^{1} ; \Lambda_{\Omega \mid\left(F \times S^{1}\right)}\right)\right) .
\end{aligned}
$$

[15, Corollary 8.6] shows that $\underline{H F}^{\infty}\left(F \times S^{1} ; \Lambda_{\Omega \mid\left(F \times S^{1}\right)}\right)=0$ for all Spin ${ }^{c}$-structures with nontrivial $H F^{+}$, so by the Künneth theorem, $H F^{\infty}\left(Y^{\prime} ; \Lambda_{\Omega \mid Y^{\prime}}\right)=0$. Using the exact sequence relating $\underline{H F^{-}}, \underline{H F}^{\infty}, \underline{H F}^{+}$, we see that

$$
\underline{H F}^{+}\left(Y^{\prime} ; \Lambda_{\Omega \mid Y^{\prime}}\right) \cong \underline{H F}^{-}\left(Y^{\prime} ; \Lambda_{\Omega \mid Y^{\prime}}\right)
$$


where we are implicitly considering only $\operatorname{Spin}^{c}$ structures which do not violate the adjunction inequality. By (3) and the Künneth theorem, we have

$$
\underline{H F}^{-}\left(Y^{\prime} ; \Lambda_{\Omega \mid Y^{\prime}}\right) \cong \underline{H F}^{-}\left(Y ; \Lambda_{\Omega \mid Y}\right) \otimes_{\Lambda[U]} \Lambda \oplus \operatorname{Tor}_{\Lambda[U]}\left(\underline{H F}-\left(Y ; \Lambda_{\Omega \mid Y}\right), \Lambda\right),
$$

which shows that the $U$-action on $\underline{H F}-\left(Y^{\prime} ; \Lambda_{\Omega \mid Y^{\prime}}\right)$, and hence on $\underline{H F^{+}}\left(Y^{\prime} ; \Lambda_{\Omega \mid Y^{\prime}}\right)$, is zero (note that the Tor term can be identified with $\underline{\widehat{H F}}\left(Y, \Lambda_{\Omega \mid Y}\right)$ which has trivial $U$-action, by definition).

It now follows from (2) that the $\Lambda[U]$-module map

$$
\underline{F_{W_{2}, \Omega_{2}}^{+}}: \underline{H F}^{+}\left(Y^{\prime} ; \Lambda_{\Omega \mid Y^{\prime}}\right) \rightarrow \underline{H F}^{+}\left(Y ; \Lambda_{\omega}\right)
$$

is surjective, so the $U$-action on $\underline{H F}^{+}\left(Y ; \Lambda_{\omega}\right)$ is also zero. This finishes the proof of Lemma 3.2.

\section{References}

[1] Y Ai, Y Ni, Two applications of twisted Floer homology, Int. Math. Res. Not. (2009) 3726-3746 MR2539188

[2] Y Ai, T Peters, The twisted Floer homology of torus bundles, Algebr. Geom. Topol. 10 (2010) 679-695

[3] D Bar-Natan, On Khovanov's categorification of the Jones polynomial, Algebr. Geom. Topol. 2 (2002) 337-370 MR1917056

[4] MJ Dunwoody, An equivariant sphere theorem, Bull. London Math. Soc. 17 (1985) 437-448 MR806009

[5] E Eftekhary, Floer homology and existence of incompressible tori in homology spheres arXiv: 0807.2326

[6] S Eliahou, L H Kauffman, M B Thistlethwaite, Infinite families of links with trivial Jones polynomial, Topology 42 (2003) 155-169 MR1928648

[7] M Freedman, J Hass, P Scott, Least area incompressible surfaces in 3-manifolds, Invent. Math. 71 (1983) 609-642 MR695910

[8] D Gabai, Foliations and the topology of 3-manifolds, J. Differential Geom. 18 (1983) 445-503 MR723813

[9] P Ghiggini, Knot Floer homology detects genus-one fibred knots, Amer. J. Math. 130 (2008) 1151-1169 MR2450204

[10] J E Grigsby, S Wehrli, On the colored Jones polynomial, sutured floer homology, and knot floer homology, Adv. Math. 223 (2010) 2114-2165

[11] A Hatcher, Algebraic topology, Cambridge Univ. Press (2002) MR1867354 
[12] M Hedden, Khovanov homology of the 2-cable detects the unknot, Math. Res. Lett. 16 (2009) 991-994 MR2576686

[13] M Hedden, L Watson, Does Khovanov homology detect the unknot? arXiv: 0805.4423

[14] U Hirsch, W D Neumann, On cyclic branched coverings of spheres, Math. Ann. 215 (1975) 289-291 MR0375321

[15] S Jabuka, T E Mark, Product formulae for Ozsváth-Szabó 4-manifold invariants, Geom. Topol. 12 (2008) 1557-1651 MR2421135

[16] M Khovanov, A categorification of the Jones polynomial, Duke Math. J. 101 (2000) 359-426 MR1740682

[17] M Khovanov, Patterns in knot cohomology. I, Experiment. Math. 12 (2003) 365-374 MR2034399

[18] P Kronheimer, T Mrowka, Khovanov homology is an unknot-detector arXiv: 1005.4346

[19] ES Lee, An endomorphism of the Khovanov invariant, Adv. Math. 197 (2005) 554-586 MR2173845

[20] W H Meeks, III, P Scott, Finite group actions on 3-manifolds, Invent. Math. 86 (1986) 287-346 MR856847

[21] W H Meeks, III, L Simon, S T Yau, Embedded minimal surfaces, exotic spheres, and manifolds with positive Ricci curvature, Ann. of Math. (2) 116 (1982) 621-659 MR678484

[22] Y Ni, Non-separating spheres and twisted Heegaard Floer homology arXiv: 0902.4034

[23] Y Ni, Knot Floer homology detects fibred knots, Invent. Math. 170 (2007) 577-608 MR2357503

[24] Y Ni, Heegaard Floer homology and fibred 3-manifolds, Amer. J. Math. 131 (2009) 1047-1063 MR2543922

[25] P Ozsváth, Z Szabó, Absolutely graded Floer homologies and intersection forms for four-manifolds with boundary, Adv. Math. 173 (2003) 179-261 MR1957829

[26] P Ozsváth, Z Szabó, Holomorphic disks and genus bounds, Geom. Topol. 8 (2004) 311-334 MR2023281

[27] P Ozsváth, Z Szabó, Holomorphic disks and knot invariants, Adv. Math. 186 (2004) 58-116 MR2065507

[28] P Ozsváth, Z Szabó, Holomorphic disks and three-manifold invariants: properties and applications, Ann. of Math. (2) 159 (2004) 1159-1245 MR2113020

[29] P Ozsváth, Z Szabó, Holomorphic disks and topological invariants for closed threemanifolds, Ann. of Math. (2) 159 (2004) 1027-1158 MR2113019 
[30] P Ozsváth, Z Szabó, Heegaard Floer homology and contact structures, Duke Math. J. 129 (2005) 39-61 MR2153455

[31] P Ozsváth, Z Szabó, On Heegaard diagrams and holomorphic disks, from: "European Congress of Mathematics", Eur. Math. Soc., Zürich (2005) 769-781 MR2185780

[32] P Ozsváth, Z Szabó, On the Heegaard Floer homology of branched double-covers, Adv. Math. 194 (2005) 1-33 MR2141852

[33] P Ozsváth, Z Szabó, Holomorphic triangles and invariants for smooth four-manifolds, Adv. Math. 202 (2006) 326-400 MR2222356

[34] P Ozsváth, Z Szabó, An introduction to Heegaard Floer homology, from: "Floer homology, gauge theory, and low-dimensional topology", (D A Ellwood, P Ozsváth, A I Stipsicz, Z Szabó, editors), Clay Math. Proc. 5, Amer. Math. Soc. (2006) 3-27 MR2249247

[35] P Ozsváth, Z Szabó, Holomorphic disks, link invariants and the multi-variable Alexander polynomial, Algebr. Geom. Topol. 8 (2008) 615-692 MR2443092

[36] P Ozsváth, Z Szabó, Link Floer homology and the Thurston norm, J. Amer. Math. Soc. 21 (2008) 671-709 MR2393424

[37] J Rasmussen, Khovanov homology and the slice genus, to appear in Invent. Math. arXiv:math.GT/0402131

[38] A Shumakovitch, Torsion of the Khovanov homology arXiv:math.GT/0405474

[39] E H Spanier, Algebraic topology, McGraw-Hill, New York (1966) MR0210112

[40] M Thistlethwaite, Links with trivial Jones polynomial, J. Knot Theory Ramifications 10 (2001) 641-643 MR1831681

[41] H Zieschang, Finite groups of mapping classes of surfaces, Lecture Notes in Math. 875, Springer, Berlin (1981) MR643627

Department of Mathematics, Michigan State University

East Lansing, MI 48824

Department of Mathematics, Caltech

Pasadena, CA 91125

mhedden@math.msu.edu, yini@caltech.edu

http://www . math.msu.edu/ mhedden/Site/Home.html, http://www.its.caltech.edu/ yini/

Proposed: Ron Fintushel

Seconded: Peter Ozsváth, Ron Stern

Received: 11 August 2009

Revised: 26 May 2010 